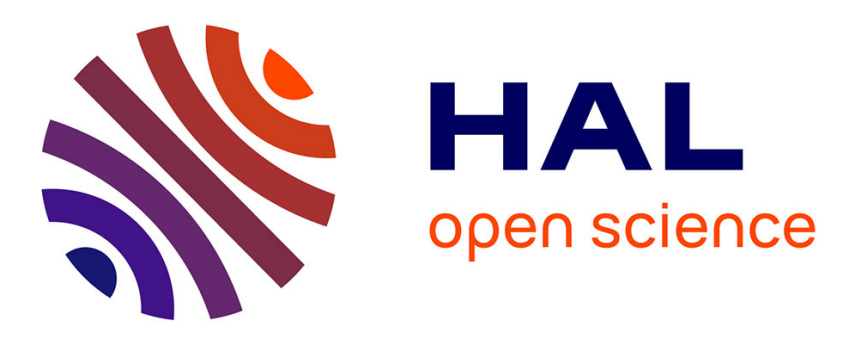

\title{
Possible charge order structure of stoichiometric $\mathrm{YbFe} 2 \mathrm{O} 4$
}

\author{
Kosuke Fujiwara, Tomoyuki Karasudani, Mamoru Fukunaga, Hiroyuki \\ Kobayashi, Jun Kano, Pierre-Eymeric Janolin, Jean-Michel Kiat, Yoshio \\ Nogami, Ryusuke Kondo, Naoshi Ikeda
}

\section{To cite this version:}

Kosuke Fujiwara, Tomoyuki Karasudani, Mamoru Fukunaga, Hiroyuki Kobayashi, Jun Kano, et al.. Possible charge order structure of stoichiometric YbFe2O4. Ferroelectrics, 2017, 512 (1), pp.85 - 91. 10.1080/00150193.2017.1349945 . hal-01779580

\section{HAL Id: hal-01779580 \\ https://hal.science/hal-01779580}

Submitted on 28 Feb 2020

HAL is a multi-disciplinary open access archive for the deposit and dissemination of scientific research documents, whether they are published or not. The documents may come from teaching and research institutions in France or abroad, or from public or private research centers.
L'archive ouverte pluridisciplinaire HAL, est destinée au dépôt et à la diffusion de documents scientifiques de niveau recherche, publiés ou non, émanant des établissements d'enseignement et de recherche français ou étrangers, des laboratoires publics ou privés. 
Possible charge order structure of stoichiometric $\mathrm{YbFe}_{2} \mathrm{O}_{4}$

KOSUKE FUJIWARA, ${ }^{1, *}$ TOMOYUKI KARASUDANI, ${ }^{1}$ MAMORU

FUKUNAGA,${ }^{1}$ HIROYUKI KOBAYASHI, ${ }^{1}$ JUN KANO,${ }^{1,2}$ PIERRE-

EYMERIC JANOLIN,${ }^{3}$ JEAN-MICHEL KIAT, ${ }^{3,4}$ YOSHIO NOGAMI, ${ }^{1}$ RYUSUKE KONDO, ${ }^{1}$ NAOSHI IKEDA ${ }^{1}$

${ }^{1}$ Graduate School of Natural Science and Technology, Okayama University, Okayama 700-8530, JAPAN.

2 Japan Science and Technology Agency, PRESTO, Saitama, 332-0012, JAPAN

${ }^{3}$ Laboratoire Structures, Propriétés et Modélisation des Matériaux, UMR CNRS-

CentraleSupélec,Université Paris-Saclay, F-92295 Châtenay-Malabry, France

${ }^{4}$ Laboratoire Léon Brillouin, CE Saclay CNRS-UMR 12, 91991 Gif-Sur-Yvette Cedex, France

corresponding author:sc422225@s.okayama-u.ac.jp 


\section{Possible charge order structure of stoichiometric $\mathrm{YbFe}_{2} \mathrm{O}_{4}$}

We report a possible charge ordering model of triangular mixed valence material $\mathrm{YbFe}_{2} \mathrm{O}_{4}$. It was found that a crystal synthesized with high stoichiometry on iron vacancy shows new and simple extinction rule in the superlattice reflections. The Laue class of the diffraction signal is expressed as $2 / \mathrm{m}$. With the consideration of the charge neutrality and the superlattice signal of $(1 / 31 / 30)$, we derived 5 possible charge ordering models of this material.

Keywords: Electric Ferroelectricity; Charge Order; $\mathrm{YbFe}_{2} \mathrm{O}_{4}$

\section{Introduction}

Recently, magneto-electric coupling in multiferroic materials has attracted much attention since it may develop some new applications in spintronic devices [1]. Though the origins of the magnetism and ferroelectricity have independent mechanisms, their correlated interaction has long been studied to overcome their incompatibility [2]. During such efforts much attention has been focused on some unconventional type of ferroelectricity, in which the primary origin of the electric dipole is not the ionic displacement [3]. The electric polarization of electronic ferroelectric is proposed as noncentrosymmetric distribution of electrons, that is driven by the correlated interaction of electrons [4].

$\mathrm{YbFe}_{2} \mathrm{O}_{4}$ is a member of $R \mathrm{Fe}_{2} \mathrm{O}_{4}(R=\mathrm{Y}, \mathrm{Dy}, \mathrm{Ho}, \mathrm{Er}, \mathrm{Tm}, \mathrm{Yb}, \mathrm{Lu}, \mathrm{Sc}$ and $\mathrm{In})$ which is proposed as a prototype of the electronic ferroelectrics. The competing interaction of iron ions is considered to appear in doubly stacked triangular layers (socalled W-layer) in the crystal cell. The interaction of the charge, spin and orbital for $3 \mathrm{~d}$ electrons of $\mathrm{Fe}^{2+}$ and $\mathrm{Fe}^{3+}$ in the triangle lattice, realizes the possible charge ordering of $\mathrm{Fe}^{2+}$ and $\mathrm{Fe}^{3+}$, which does not have the inversion symmetry [5]. So geometrical frustration on the triangular lattice plays a key role in the ground state configuration of 
charges as well as spins.

It has been discussed that the polar charge-ordering $(\mathrm{CO})$ pattern realizes a macroscopic spontaneous ferroelectric polarization. The $\mathrm{CO}$ has been observed in diffraction and resonant X-ray scattering experiments [5-7]. Below $500 \mathrm{~K}$ twodimensional (2D) CO was found in diffuse streak line $(1 / 31 / 3 L)$ in reciprocal space of hexagonal expression. Below $330 \mathrm{~K}$ the streak line shrinks to superlattice spots $(1 / 3 \mathrm{H} / 3$ $n+1 / 2$ ), where $n$ is integer, indicating a three-dimensional (3D) charge ordering. The development of the 3D CO has been discussed in relation with the development of the macroscopic electric polarization [6-8].

However, the existence of the macroscopic polarization has not been confirmed yet. Though researchers have agreed on the existence of the charge ordering below $330 \mathrm{~K}$, a consensus about the ground state of the $\mathrm{CO}$ model have not reached. For example, it is suggested that the ground state of $\mathrm{CO}$ structure is non-polar $C 2 / m$ in the W-layer in which the CO structure was derived from the bond valence sum (BVS) calculation [8]. But we consider that the BVS analysis might contains the effect of the mixed domain information, leading some inconsistency.

Thus, the ground states of $\mathrm{CO}$ structure in $\mathrm{RFe}_{2} \mathrm{O}_{4}$ has not been established yet and still remains an open research problem [10-12]. It is known that physical property relate to $\mathrm{CO}$ structure strongly depends on the oxygen stoichiometry in $R \mathrm{Fe}_{2} \mathrm{O}_{4}[9,13$ 14]. The variation of the oxygen vacancies affects the population ratio of $\mathrm{Fe}^{2+}$ and $\mathrm{Fe}^{3+}$ in triangular lattice. Recently we found that the single crystal grown with a standard protocol contains significant amount of iron vacancies [15]. We also found that the crystal having less iron vacancy i.e., the stoichiometric sample, shows longer spin coherence and pure ferri-magnetism. 
Interestingly, the coherence of spin corresponds to that of charges, since both ordering appears on the same iron ions. Thus it is considered that a material having long spin coherence may have long CO coherence. In this paper, we report that the iron stoichiometric single crystal of $\mathrm{YbFe}_{2} \mathrm{O}_{4}$ has simpler extinction rule of the superlattice reflections, compared to the previous reports. From observation of new extinction rule, we succeeded in deriving the unit cell of the iron charge superlattice. This is the first report of the charge unit cell which was directly derived from the observation. Also from the consideration of the charge neutrality and the superlattice signal of (1/3 $1 / 30)$ in reciprocal space, we succeeded in deriving the charge ordering models in five possible patterns for the first time.

\section{Experiment}

A single crystal of $\mathrm{YbFe}_{2} \mathrm{O}_{4}$ was grown using the floating zone (FZ) melting method from a seed rod, which was a mixture of $\mathrm{Fe}_{2} \mathrm{O}_{3}$ and $\mathrm{Yb}_{2} \mathrm{O}_{3}$. To suppress iron vacancy, we changed the mixture ratio of $\mathrm{Fe}_{2} \mathrm{O}_{3} / \mathrm{Yb}_{2} \mathrm{O}_{3}$ of the seed rod. (details are described in ref [15]) The chemical components were analyzed with X-ray fluorescence (XRF) (ORBIS EDAX) and thermogravimetric analysis (TGA) (SII TG/DTA7300) for crushed samples. In XRF measurement, $\mathrm{Yb}$ / Fe ratio was estimated from the integral intensity of the Fe- Ka and $\mathrm{Yb}-L \alpha$ fluorescence line. The fluorescence intensity had been calibrated with standard samples that were mixture of $\mathrm{Fe}_{2} \mathrm{O}_{3}$ and $\mathrm{Yb}_{2} \mathrm{O}_{3}$ powders. The error of the calibration line was less than $0.2 \%$. In order to characterize oxygen stoichiometry, we carried out thermogravimetric analysis in air up to about $600^{\circ} \mathrm{C}$, at which temperature the $\mathrm{YbFe}_{2} \mathrm{O}_{4}$ sample change to $\mathrm{YbFe}_{2} \mathrm{O}_{4.5}$.

The development of the charge ordering was observed with the reciprocal space observation by oscillation photography, using X-ray imaging plate (IP) camera with $\mathrm{Cu}$ - 
Ka radiation (RIGAKU: RAPID-II Valimax-Cu). Four-axis diffractometor HUBAR512 with Mo-Ka and control software SPEC was used to measure the Bragg spots mapping. $(003)_{\mathrm{h}},(009)_{\mathrm{h}},(110)_{\mathrm{h}}$ and $(101)_{\mathrm{h}}$ spots were used to calculate UB matrix. These X-ray diffractions were measured at room temperature (RT).

\section{Results}

Table 1 . shows the chemical components of the samples measured by XRF and TGA. As shown in Table 1., we found that samples grown with the standard method (sample I) contain iron vacancies of more than $10 \%$. The samples grown with excess iron have iron vacancies less than 3\%. We estimated the oxygen concentration of all the samples from TGA and XRF data as shown in Table 1. The ratio of $\mathrm{Fe}^{2+}$ and $\mathrm{Fe}^{3+}$ was independent to $\mathrm{Fe}_{2} \mathrm{O}_{3} / \mathrm{Yb}_{2} \mathrm{O}_{3}$ ratio. We also carried out Mössbauer spectroscopy to confirm the TGA result.

Figure 1. shows the reciprocal space picture near superlattice spots $(1 / 31 / 3 L)$ taken with IP oscillation camera. The sample I has diffuse scattering along $(1 / 31 / 3 \mathrm{~L})$ where $L$ is continuous. In sample II, superlattice spots $(1 / 31 / 30.5+n)$ and incommensurate modulation $(1 / 3+\delta 1 / 3+\delta n)$ for $(\delta \sim 0.03)$ where $n$ is integer were observed. Sample II result is consistent with previous reports [7-8]. On one hand, the most stoichiometric sample (sample III) which has long magnetic coherence length showed new extinction rule expressed as $(1 / 31 / 31.5 n)$ where $n$ is integer. No incommensurate modulation was observed on this sample III.

To measure the extinction rule of sample III, we observed the reciprocal space with 4circle diffractometor. Figure 2 (a-c) shows reciprocal mapping of (1/3 1/3 L), (1/3 -2/3 $L)$ and $(2 / 3-1 / 3 L)$ of this crystal respectively. The extinction rules of $L$ were $(1 / 31 / 3$ $1.5 n),(1 / 3-2 / 30.5+1.5 n)$ and $(2 / 3-1 / 31+1.5 n)$. We also observed $(-1 / 3-1 / 3 L),(-1 / 3$ 
$2 / 3 L)$ and $(-2 / 31 / 3 L)$. These data are summarized in Figure 2(d). In the figure, the extinction rule found on this material is summarized in red, green and blue circle indicates $\mathrm{L}=1.5 n, 0.5+1.5 n$ and $1+1.5 n$, respectively. As shown in the figure, a mirror symmetry was found in the reciprocal space of this crystal (in Red line). The observed symmetries are categorized $2 / \mathrm{m}$ in Laue class. The Laue classes correspond to the eleven centrosymmetric crystallographic point groups. Considering Friedel's pair, it is difficult to distinguish by diffraction between a centrosymmetric point group and non-centrosymmetric subgroups.

\section{Discussion}

As described above, the space group and the ground state of the $\mathrm{CO}$ have not yet been established. As this stoichiometric crystal has long charge coherence, the simple extinction rule of superlattice spots demonstrated mono domain nature of charge ordering. So we analyzed this extinction rule to derive the $\mathrm{CO}$ model. It is known that the symmetry of high temperature phase is $R-3 m$. From this, the CO phase should be a subgroup of $R-3 m$. Laue class of $2 / \mathrm{m}$ consists of 22 space groups and three point groups (2, m, $2 / \mathrm{m})$. In the 22 space groups $C 2, C m$ and $C 2 / m$ are selected since they are subgroup of $R-3 m$ and compatible with the wave structure of $(1 / 31 / 30)$ in hexagonal structure. So we started to examine these three space group to derive the $\mathrm{CO}$ models.

At first, we noticed the extinction rule of space group for $C 2, C m$ and $C 2 / m$, they are $\mathrm{h}$ $+\mathrm{k}=2 n$. Adapting this extinction rule to the observed one, the base vector in the reciprocal space are derived as

$$
\left\{\begin{array}{l}
\overrightarrow{a_{m}^{*}}=\frac{1}{2} \overrightarrow{a_{h}^{*}}-\frac{1}{2} \overrightarrow{b_{h}^{*}}-\frac{1}{2} \overrightarrow{c_{h}^{*}} \\
\overrightarrow{b_{m}^{*}}=\frac{1}{6} \overrightarrow{a_{h}^{*}}+\frac{1}{6} \overrightarrow{b_{h}^{*}} \\
\overrightarrow{c_{m}^{*}}=\frac{3}{2} \overrightarrow{c_{h}^{*}}
\end{array}\right.
$$


Here, the reciprocal lattice is denoted by base vectors as $\overrightarrow{a_{h}^{*}}, \overrightarrow{b_{h}^{*}}, \overrightarrow{c_{h}^{*}}$ in hexagonal setting. Then we can obtain the lattice constants from the base vectors in reciprocal space as given

$$
\left\{\begin{array}{l}
\overrightarrow{a_{m}}=\overrightarrow{a_{h}}-\overrightarrow{b_{h}} \\
\overrightarrow{b_{m}}=3 \overrightarrow{a_{h}}+3 \overrightarrow{b_{h}} \\
\overrightarrow{c_{m}}=\frac{1}{3} \overrightarrow{a_{h}}-\frac{1}{3} \overrightarrow{b_{h}}-\frac{2}{3} \overrightarrow{c_{h}}
\end{array}\right.
$$

Where, $\overrightarrow{a_{h}}, \overrightarrow{b_{h}}, \overrightarrow{c_{h}}$ is the unit cell vectors in hexagonal setting.

The lattice constants derived uniquely were $\mathrm{a}=5.984 \AA, \mathrm{b}=10.365 \AA, \mathrm{c}=16.702 \AA$, $\alpha=\gamma=90, \beta=96.80$, which indicates a monoclinic unit cell. This is the unit cell of the charge ordered superlattice.

Next, we check the symmetry operation. $C 2$ has 2 -fold rotation in $0 y 0$, and $C m$ has mirror in $x, 0, z$. And $C 2 / m$ has both symmetry operations. From these restriction, 2 type of the unit cell are derived as shown in Figure 3. One unit cell has its center at the center of U-layer. Another cell locates it at the center of W-layer.

Then we consider the possible $\mathrm{CO}$ models by setting $\mathrm{Fe}^{2+}$ and $\mathrm{Fe}^{3+}$ on the $\mathrm{Fe}$-site in these unit cells. In order to build the charge order model, we considered three conditions as follows, 1) the asymmetric minimum unit in $C 2, C m$ and $C 2 / m$ symmetry has 6,8 and 4 iron positions, respectively. 2) And the charge neutrality requires to put equal amount of $\mathrm{Fe}^{2+}$ and $\mathrm{Fe}^{3+}$ into the sites. 3) The arrangement of $\mathrm{Fe}^{2+}$ and $\mathrm{Fe}^{3+}$ need to form the wave structure of $(1 / 31 / 30)$ in reciprocal space. Considering these conditions 24 possible $\mathrm{CO}$ models were derived. ( $\mathrm{Cm}$ has 4 patterns, $\mathrm{C} 2$ has 18 patterns and $\mathrm{C} 2 / \mathrm{m}$ has 2 patterns)

In these $24 \mathrm{CO}$ models, there are some identical patterns. Finally, 5 independent $\mathrm{CO}$ 
models were derived as shown in Figure 4. We mention that the electric polarization is found in all but one model, as demonstrated in Table.2. The A-C models have a local electric polarization in the $\mathrm{W}$-layer. Only model A has a macroscopic electric polarization along $c$-axis in hexagonal system. The model B has a macroscopic electric polarization along $\left[\begin{array}{lll}1 & 1 & 0\end{array}\right]_{\mathrm{h}}$. The model $\mathrm{C}$ is anti-ferroelectric and does not have a macroscopic electric polarization. On one hand, models D, E have no local electric polarization in $\mathrm{W}$-layer. The model $\mathrm{D}$ has a macroscopic electric polarization along $\left[\begin{array}{ll}1 & 1\end{array}\right.$ 0] $]_{\mathrm{h}}$. The model $\mathrm{E}$ has no electric polarization. The $\mathrm{E}$ is identical to the model proposed by Groot et al. [8].

Thus, the simple extinction rule of the superlattice spots found in the iron stoichiometric sample leads to 5 possible models for the ground state of the charge ordering. This result shows the importance of chemical stoichiometry, especially for the iron ion. This is the first report on the $\mathrm{CO}$ model of $R \mathrm{Fe}_{2} \mathrm{O}_{4}$, which is derived from the direct observation of superlattice extinction rule. It is considered that the complex extinction rule as reported in previous reports, like $(1 / 31 / 30.5+n)$ where $n$ is integer, might be come from the mixed state of $\mathrm{CO}$ domain. In order to narrow down these $5 \mathrm{CO}$ models to one exact model, some experimental approaches are possible. One is the direct observation of electric polarization. If the $\mathrm{CO}$ structure belong to space group $\mathrm{Cm}$ or $C 2$, this material is ferroelectric, which can be distinguished with P-E hysteresis loop or pyro-electric current observation. Another possible approach is the direct observation of the $\mathrm{CO}$ model with the combined method of diffraction experiment and Mössbauer spectroscopy, which possibly can distinguish the structure factor and the contribution of each iron ions. Now we are starting a trial experiment of the Mössbauer diffraction using synchrotron light source at BL11XU in Spring-8 (2015B3511). 


\section{Summary}

We derived five possible $\mathrm{CO}$ models of triangular iron charge order material $\mathrm{YbFe}_{2} \mathrm{O}_{4}$. A crystal having mono domain state of charge ordering was synthesized with suppressing the iron vacancy. A highly stoichiometric sample has a new and simple extinction rule of the superlattice signal as (1/3 1/3 1.5n), where $n$ is integer, and the signal has Laue class of $2 / m$. Considering the reflection condition, we derived lattice constants and 5 possible $\mathrm{CO}$ models deductively for the first time.

\section{Acknowledgements}

The Program for Advancing Strategic International Networks to Accelerate the Circulation of Talented Researchers from JSPS. The stay of Kosuke Fujiwara in CentraleSupélec has been supported by Erasmus Mundus EASED programme (Grant 2012-5538/004-001) coordinated by CentraleSupélec.

\section{References}

[1] Hans. Schmid: Multi-ferroic magnetoelectrics. Ferroelectrics 1994;162.1: 317338.

[2] Nicola A. Hill: Why are there so few magnetic ferroelectrics? The Journal of Physical Chemistry B 2000;104.29: 6694-6709.

[3] O. Prokhnenko, R. Feyerherm, M. Mostovoy, N. Aliouane, E. Dudzik, A. U. B. Wolter, A. Maljuk, and D. N. Argyriou: Coupling of frustrated Ising spins to the magnetic cycloid in multiferroic $\mathrm{TbMnO}_{3}$. Physical review letters 2007;99(17): 177206.

[4] S. Ishihara: Electronic ferroelectricity and frustration. Journal of the Physical Society of Japan 2010;79(1): 011010.

[5] N. Ikeda, H. Ohsumi, K. Ohwada, K. Ishii, T. Inami, K. Kakurai, and H. Kitô: Ferroelectricity from iron valence ordering in the charge-frustrated system $\mathrm{LuFe}_{2} \mathrm{O}_{4}$. Nature 2005;436(7054): 1136-1138. 
[6] J. de Groot, K. Marty, M. D. Lumsden, A. D. Christianson, S. E. Nagler, S. Adiga, and R. de Souza: Competing ferri-and antiferromagnetic phases in geometrically frustrated $\mathrm{LuFe}_{2} \mathrm{O}_{4}$. Physical review letters 2012;108(3): 0372061.

[7] Y. Yamada, K. Kitsuda, S. Nohdo, and N. Ikeda: Charge and spin ordering process in the mixed-valence system $\mathrm{LuFe}_{2} \mathrm{O}_{4}$ : Charge ordering. Physical Review B 2000;62(18): 12167.

[8] J. de Groot, T. Mueller, R. A. Rosenberg, D. J. Keavney, Z. Islam, J. W. Kim, and M. Angst: Charge order in $\mathrm{LuFe}_{2} \mathrm{O}_{4}$ : an unlikely route to ferroelectricity. Physical review letters 2012;108(18): 187601.

[9] M. Angst: Ferroelectricity from iron valence ordering in rare earth ferrites?, physica status solidi (RRL)-Rapid Research Letters 2013;7(6): 383-400.

[10] N. Ikeda, T. Nagata, J. Kano, and S. Mori: Present status of the experimental aspect of $R \mathrm{Fe}_{2} \mathrm{O}_{4}$ study. Journal of Physics: Condensed Matter 2015;27(5): 053201.

[11] M. Iwata and Y. Ishibashi: Possible commensurate phases in $\mathrm{LuFe}_{2} \mathrm{O}_{4}$. Journal of the Physical Society of Japan 2012 ;81(3): 035003.

[12] F. Wang, J. Kim, G. D. Gu, Y. Lee, S. Bae and Y. J. Kim: Oxygen stoichiometry and magnetic properties of $\mathrm{LuFe}_{2} \mathrm{O}_{4+\delta}$. Journal of Applied Physics 2013;113(6): 063909.

[13] Y. Horibe, K. Yoshii, N. Ikeda and S. Mori: Oxygen-deficient effect on charge ordering in spin-and charge-frustrated ferrite $\mathrm{YFe}_{2} \mathrm{O}_{4-\delta}$. Physical Review B 2009;80(9): 092104.

[14] J. Bourgeois, M. Hervieu, M. Poienar, A. M. Abakumov, E. Elkaim, M. T. Sougrati and A. Maignan: Evidence of oxygen-dependent modulation in $\mathrm{LuFe}_{2} \mathrm{O}_{4}$. Physical Review B 2012;85(6): 064102.

[15] K. Fujiwara, M. Miyajima, M. Fukunaga, J. Kano, H. Kobayashi and N. Ikeda: Iron vacancy effect on the magnetization of $\mathrm{YbFe}_{2} \mathrm{O}_{4}$. Transactions of the Materials Research Society of Japan 2016;41(1): 139-142. 
Table 1. Chemical stoichiometry of samples.

\begin{tabular}{crrr}
\hline Sample & $\mathrm{Fe}_{2} \mathrm{O}_{3} / \mathrm{Yb}_{2} \mathrm{O}_{3}$ ratio & $\mathrm{Fe} / \mathrm{Yb}$ ratio by XRF & Average of Fe valence \\
\hline I & 2.00 & $1.79 \pm 0.02$ & 2.541 \\
II & 2.05 & $1.85 \pm 0.02$ & 2.584 \\
III & 2.20 & $1.95 \pm 0.02$ & 2.523 \\
\hline
\end{tabular}


Table 2. Electric polarization and space group of $\mathrm{CO}$ models.

\begin{tabular}{cccc}
\hline CO model & Space group & Direction of polarization & Local polarization in W-layer \\
\hline $\mathrm{A}$ & $\mathrm{Cm}$ & {$\left[\begin{array}{llll}1 & -1 & 2\end{array}\right]_{\mathrm{h}}$} & $\cap$ \\
$\mathrm{B}$ & $\mathrm{C} 2$ & {$\left[\begin{array}{lll}1 & 1 & 0\end{array}\right]_{\mathrm{h}}$} & \\
$\mathrm{C}$ & $\mathrm{C} 2 / \mathrm{m}$ & None & $\times$ \\
$\mathrm{D}$ & $\mathrm{C} 2$ & {$\left[\begin{array}{lll}1 & 1 & 0\end{array}\right]_{\mathrm{h}}$} & $\times$ \\
$\mathrm{E}$ & $\mathrm{C} 2 / \mathrm{m}$ & None & \\
\hline
\end{tabular}


Figure 1. The reciprocal lattice mapping of each samples by X-ray oscillation photography. Sample I shows diffused line. Sample II shows conventional reflection described as $(1 / 31 / 30.5+n)$. Sample III has a new simpler extinction rule described as $(1 / 31 / 31.5 n)$

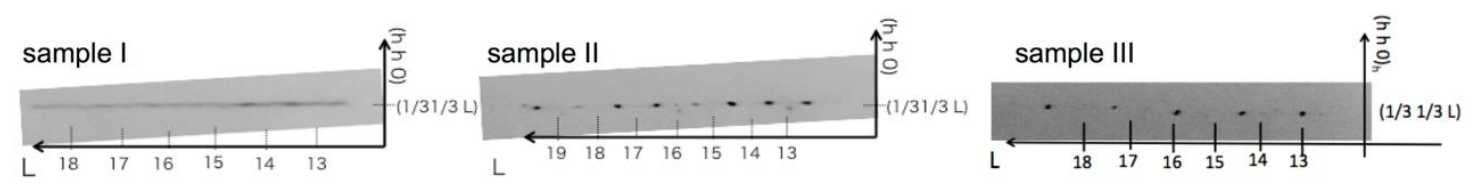


Figure 2. The extinction rule of sample III in reciprocal space. Fig (a-c) is line scans for $(1 / 31 / 3 L),(1 / 3-2 / 3 L)$ and $(2 / 3-1 / 3 L)$, respectively, detected with a four-circle diffractometer. (d) Summary of the observed extinction rules depicted on $a^{*}-b^{*}$ plane. Red, green and blue circle indicate $\mathrm{L}$ component of $(1 / 31 / 3 \mathrm{~L})$ scan for $\mathrm{L}=1.5 n, 0.5+$ $1.5 n$ and $1+1.5 n$, respectively. A mirror symmetry in reciprocal space is indicated in Red line.

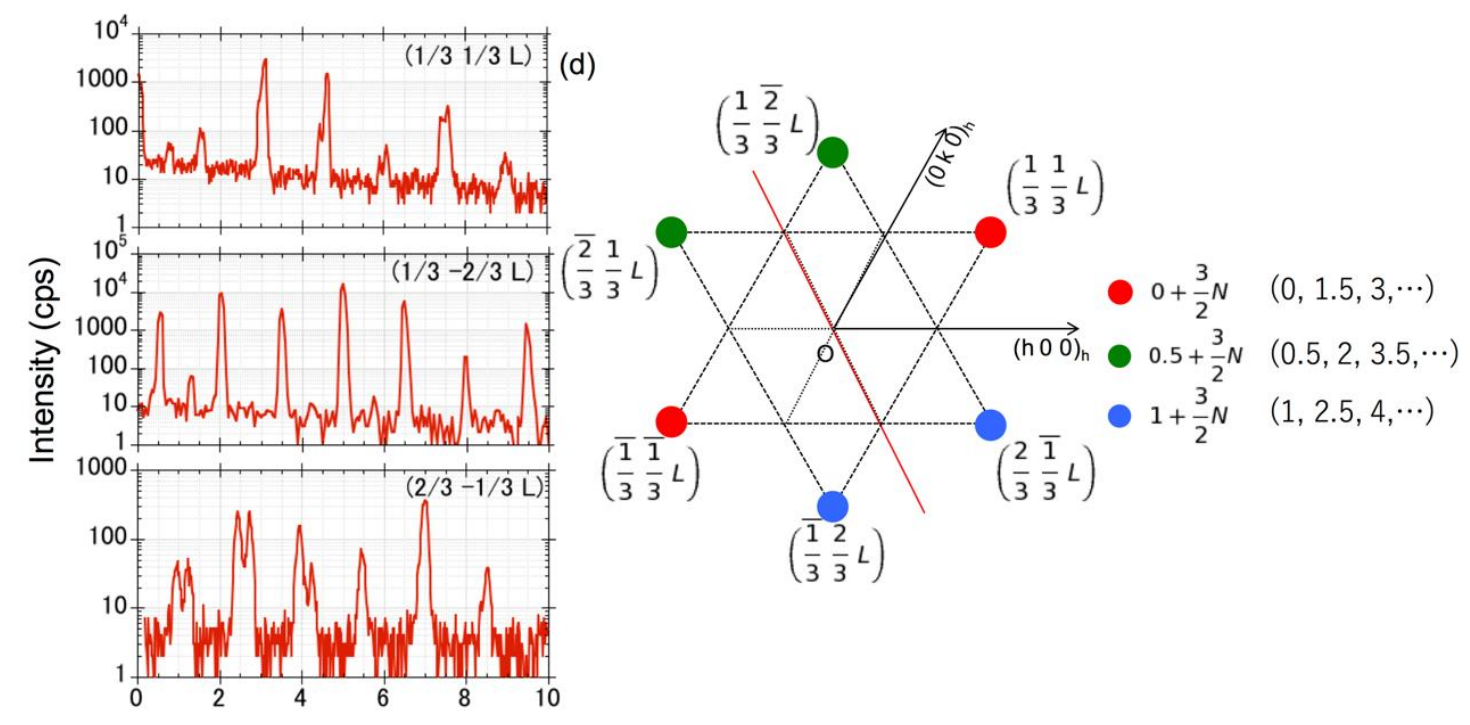


Figure 3. The derived monoclinic unit cells. (a) Unit cell center is located at the center of $\mathrm{Yb}$ layer (so-called U-layer). (b) Unit cell center is located at the center of W-layer.

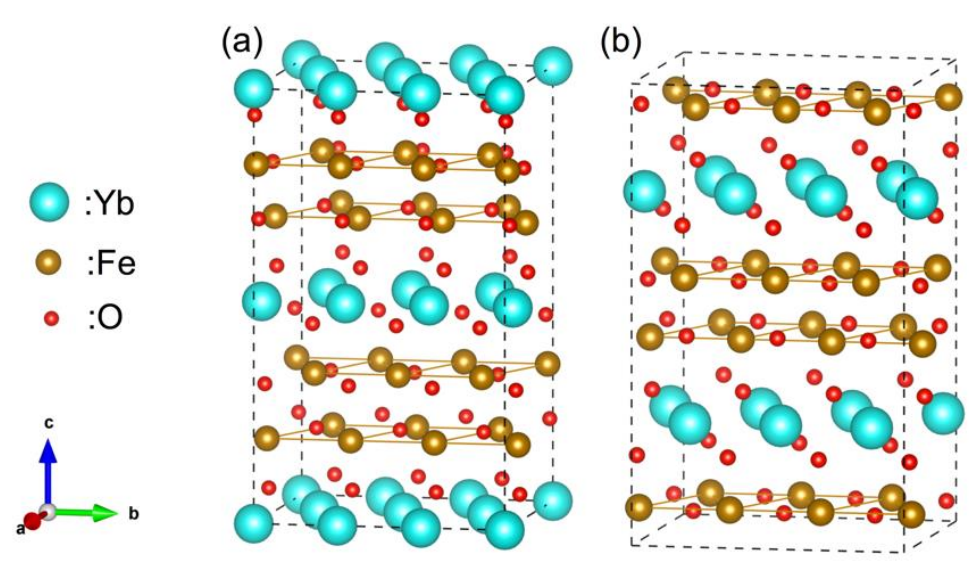


Figure 4. The CO models we derived. The blue block vector represents a local electric polarization.

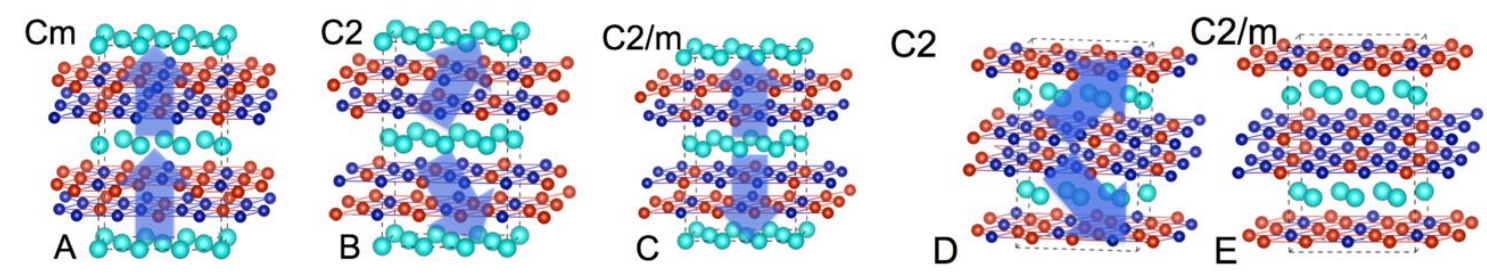

